\title{
Quality Measurement of Unwrapped Three-dimensional Fingerprints: a Neural Networks Approach
}

\author{
Ruggero Donida Labati, IEEE, Member, Angelo Genovese, IEEE, Member, \\ Vincenzo Piuri, IEEE, Fellow, Fabio Scotti, IEEE, Member \\ Department of Information Technology \\ Università degli Studi di Milano \\ Milano, 20122, Italy. \\ \{ruggero.donida, angelo.genovese, vincenzo.piuri, fabio.scotti\}@unimi.it
}

\begin{abstract}
Traditional biometric systems based on the fingerprint characteristics acquire the biometric samples using touch-based sensors. Some recent researches are focused on the design of touch-less fingerprint recognition systems based on CCD cameras. Most of these systems compute three-dimensional fingertip models and then apply unwrapping techniques in order to obtain images compatible with biometric methods designed for images captured by touch-based sensors. Unwrapped images can present different problems with respect to the traditional fingerprint images. The most important of them is the presence of deformations of the ridge pattern caused by spikes or badly reconstructed regions in the corresponding three-dimensional models. In this paper, we present a neural-based approach for the quality estimation of images obtained from the unwrapping of three-dimensional fingertip models. The paper also presents different sets of features that can be used to evaluate the quality of fingerprint images. Experimental results show that the proposed quality estimation method has an adequate accuracy for the quality classification. The performances of the proposed method are also evaluated in a complete biometric system and compared with the ones obtained by a well-known algorithm in the literature, obtaining satisfactory results.
\end{abstract}

\section{INTRODUCTION}

Most of the fingerprint recognition systems require that the finger touches a sensor in order to acquire the biometric sample. In the literature, there are also less-constrained recognition systems based on fingertip images captured using CCD cameras and without touching a sensor. The possibility to perform the biometric recognition by using sample acquisition techniques that do not require a contact with a sensor introduces a great value. In fact, it allows the deployment of these systems in a wider range of applicative contexts, increases the privacy compliancy of the recognition techniques, increases the acceptability of the users, removes problems related to nonlinear distortions caused by different pressures of the fingers on the sensors, and reduces the sensibility to the dust present on the sensors. Considering that touch-less recognition systems based on a single camera can produce images affected by perspective distortions, many systems merge the information obtained by more cameras or projected patterns in order to compute three-dimensional models of the fingers, which do not present any kinds of deformations. Usually, the obtained models are then converted in bidimensional images in order to perform the biometric recognition by using well-known methods designed for traditional fingerprint images. This step is called unwrapping or unrolling.

The fingertip images captured using touch-less sensors are very different from the images captured using traditional sensors. These images, in fact, present more noise, can be affected by strong reflections, and present a more complex background. Considering that the biometric recognition process is based only on the information related to the pattern composed by ridges and valleys, the skin of the finger should also be considered as a part of the background. In particularly unconstrained setups, there can also be present out of focus and motion blur problems. Moreover, the quality of the obtained samples is strictly related to the applicative contexts and to the ability of the users. For these reasons, a correct biometric acquisition is more difficult to be performed in touch-less systems.

Considering biometric systems that compute threedimensional fingertip models, incorrect sample acquisitions can drastically reduce the visibility of the ridge pattern, causing problems during the three-dimensional reconstruction step. For example, biometric systems that compute threedimensional models by using $\mathrm{N}$-view techniques [1] reconstruct the finger volume by searching points that match in the images obtained by the different views. If the ridge pattern is not properly visible in the captured images, the estimation of the matching points can produce erroneously matched pairs of points, resulting in artifacts in the threedimensional models. Consequently, the presence of artifacts in the fingertip models can produce deformations in the unwrapped images.

The images obtained by the unwrapping of threedimensional fingertip models can present different problems:

1) deformations of the images caused by badly reconstructed portions of the three-dimensional model;

2) artifacts caused by the presence of spikes in the threedimensional model;

3) areas with artifacts created during the enhancement of 
(a)

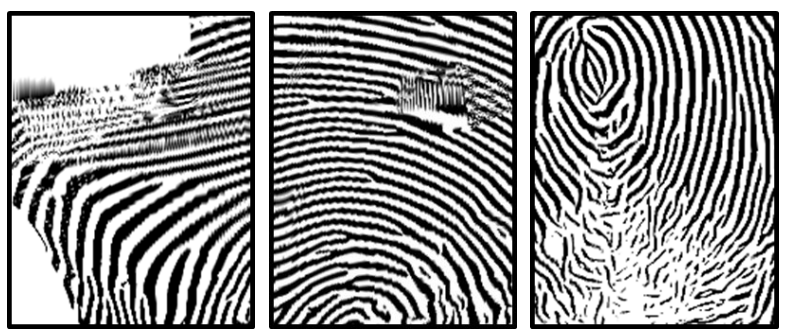

Fig. 1. Examples of problems that can be present in unwrapped fingerprint images: (a) a deformation caused by a badly reconstructed portion of the three-dimensional model; (b) an artifact caused by the presence of a spike in the three-dimensional model; (c) an area with low visibility of the ridge pattern.

the fingertip pattern and corresponding to low-contrast regions of the input images.

Fig. 1 shows an example of these problems.

Images affected by deformations and artifacts can drastically reduce the accuracy of the biometric recognition systems. For this reason, the use of quality estimation methods is necessary to improve the final accuracy and reliability of the whole biometric system.

In the literature, there are many quality estimation techniques designed for touch-based fingerprint recognition systems [2]. There are also studies regarding the quality of touchless fingertip images [3], but, at the best of our knowledge, there are no methods specifically designed for the evaluation of unwrapped images obtained from three-dimensional models.

This paper presents an approach specifically designed for the quality evaluation of unwrapped fingerprint images. The contribution of the paper is twofold: we propose a specific set of features usable for the quality evaluation of these particular images, and we describe the results obtained by applying the proposed approach in a complete biometric system.

The schema of the proposed approach is shown in Fig. 2. First, a three-dimensional model and an unwrapped image are computed by using the methods described in [1]. The obtained fingerprint image is then used to extract a set of distinctive features. Finally, a neural classifier estimates the quality of the unwrapped fingerprint image.

The results obtained by the classification step are compared with the ones obtained by applying a well-known method in the literature for the quality classification of fingerprint images captured by touch-based sensors. The obtained results are then used to evaluate the improvement of accuracy obtained by using the proposed approach in a complete biometric recognition system.

The paper is structured as follows. Section II proposes a brief literature review regarding the quality evaluation of touch-based and touch-less fingerprint images. Section III describes the proposed feature extraction and classification methods. Section IV presents the results obtained by using different sets of features and by applying the proposed approach in a complete biometric system. Finally, conclusions and future works are proposed in section $\mathrm{V}$.

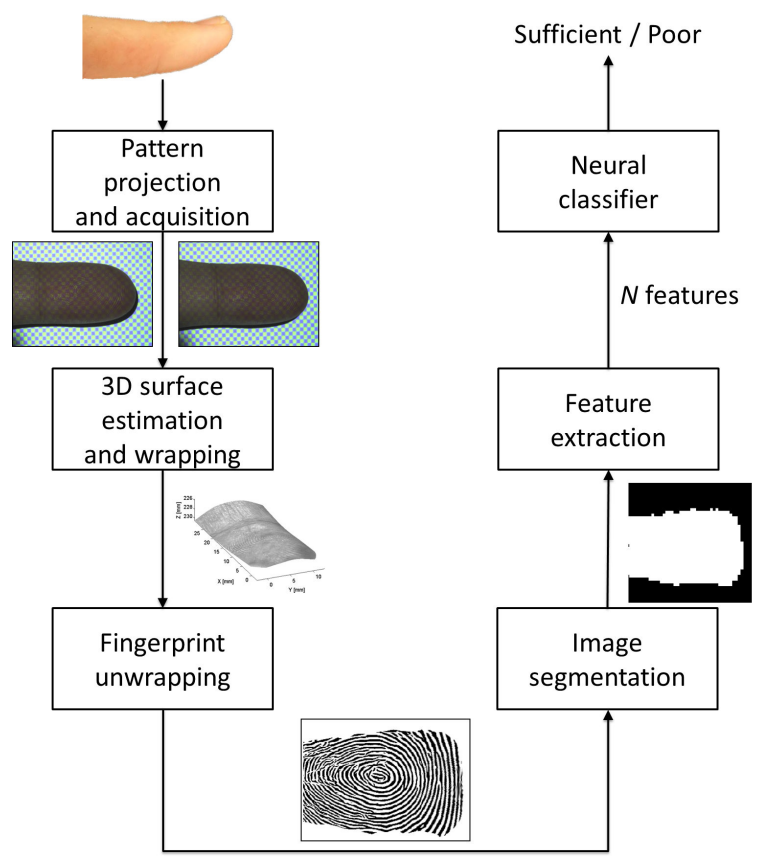

Fig. 2. Schema of the proposed approach.

\section{RELATED WORKS}

In the literature, there are methods for the reconstruction of three-dimensional fingertip models based on different technologies: a method presented in [4] uses an illuminator shaped as a ring-mirror, the method described in [5] is based on shape-from silhouette technique and uses five cameras placed around the finger, the method treated in $[6,7]$ is based on the projection of a sinusoidal pattern, and the reconstruction technique proposed in [1] is based the information obtained by using a two-view system and a static projected pattern.

Most of the current biometric recognition systems based on fingertip three-dimensional models map the reconstructed model in a bidimensional space, obtaining an unwrapped image. Unwrapped images are then used to perform identity comparisons based on traditional methods designed for touchbased fingerprint recognition systems. An approach for the computation of unwrapped fingerprint images that approximates the finger shape by a cylindrical model is described in [8]. Another approach described in [8] can be considered as non-parametric and uses local distance measures to preserve the relations between the points of the model. The technique described in [6] is based on the fitting of multiple circles to the finger model and maps each fitted circle into the bidimensional space. The method presented in [9] computes a conversion of the three-dimensional model in spherical coordinates and then performs a refinement step. A local approach is described in [10] and is based on the computation of local fitting planes, minimizing a cost function that describes the movement exerted between each point and its neighbors. The technique presented in [11] considers also the distortion due to the finger pressure on the sensor in order to obtain unwrapped images 
compatible with traditional contact-based fingerprint images.

The quality analysis of biometric samples is very important in biometric systems because it permits to discard the noisy or bad captured samples. In the literature, there are quality evaluation techniques designed for touch-less fingertip images [3] and there are many methods for the evaluation of touchbased fingerprint samples [2].

Many quality evaluation techniques designed for touchbased fingerprint images are based on the evaluation of local features. The method described in [12] evaluates the probability density function (PDF) of local regions. The technique presented in in [13] computes the used features by applying a set of Gabor filters with different orientations to the local areas of the image. Other techniques are based on global characteristics. For example, the method described in [14] is based on the evaluation of the energy distribution rate in wavelet compressed fingerprint images. The technique proposed in [15] combines local and global features related to the frequency domain (a ring structure of DFT magnitude and directional Gabor features) and to the spatial domain (black pixel ratio of the central area). Also the method described in [16] combines local and global characteristics and is based on features related to the effective area, energy concentration, spatial consistency, and directional contrast.

One of the most used quality estimation techniques in the literature is described in [17]. This technique is based on neural networks and classifies five levels of quality, from poor to excellent. The method is specifically designed for working in fingerprint recognition systems based on identity comparison algorithms that use minutiae features, like the one presented in [18].

At the best of our knowledge, in the literature there are no quality estimation techniques specifically designed for fingerprint images obtained by unwrapping three-dimensional models.

\section{The PROPOSED APPROACH}

The proposed approach aims to evaluate the presence of deformations in unwrapped fingerprint images and to accurately classify the quality of these images. The quality can be expressed by using real or discrete values. In this approach, we use discrete classes in order to easily discard low quality biometric samples during the recognition process.

The unwrapped images and the related three-dimensional models treated by this approach are obtained by using the method described in [1]. The three-dimensional reconstruction method is based on a single two view acquisition and a static projected pattern. After a segmentation of the unwrapped fingerprint image, the proposed approach extracts different sets of features, which are related to the shape of the region of interest (ROI), the number and quality of the minutiae, and the local characteristics of the ridge pattern. The quality estimation is then performed by using neural classifiers.

\section{A. Three-dimensional models and unwrapped images}

The technique used to obtain unwrapped fingerprint images is described in [1]. The acquisition setup is composed by two (a)

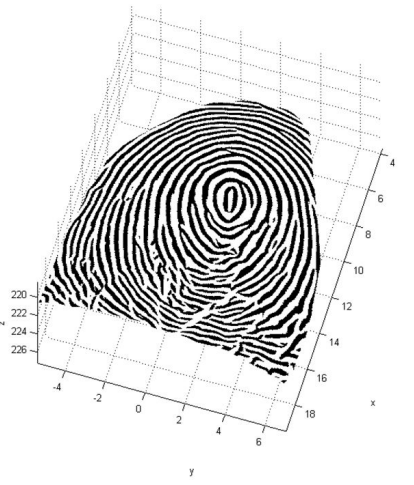

(b)

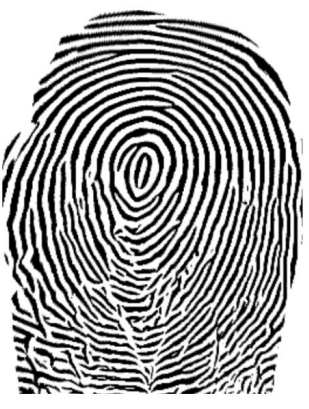

Fig. 3. Example of a three-dimensional finger model and the corresponding unwrapped image: (a) three-dimensional model; (b) resulting unwrapped image.

calibrated CCD cameras and a DLP projector. This technique can be divided in the sequent steps.

- Pattern projection and acquisition: a static pattern composed by blue and green squares is computed and projected on the finger.

- Image preprocessing: an image describing the fingertip and an image representing the projected pattern are extracted from every captured image.

- Extraction and matching of the reference points: the centroids of the projected squares visible in the left view image are matched with the ones visible in the right view image by using an algorithm based on the calibration data and on the correlation between local areas of the fingertip images. The obtained result consists in a vector of pairs of points that correspond in the two views.

- Three-dimensional surface estimation and image wrapping: considering the obtained pairs of corresponding points and the data related to the system calibration, the volume of the finger is estimated and the fingertip texture is then wrapped on the three-dimensional model.

- Texture enhancement: the visibility of the ridge pattern is enhanced by using an algorithm based on Gabor filters, which is similar to the one described in [3, 19].

- Fingertip unwrapping: similarly to the algorithm described in [6], the used technique considers the finger shape as composed by a set of rings with different diameters. The unwrapped image is obtained by converting the estimated circles in Cartesian coordinates and by removing the outliers. Figure 3 shows an example of a three-dimensional model and the corresponding unwrapped image.

\section{B. Image segmentation}

The image $I_{R}$ representing the ROI is computed by applying an empirically estimated threshold $t_{s}$ to local standard deviation values related to $l \times l$ squared regions. 


\section{Feature extraction}

Different sets of features related to different image characteristics are extracted.

- Features related to the minutiae $\left(F_{M}\right)$ : the number of minutiae points, and the mean and standard deviation of their quality are computed. The method used for the extraction of the minutiae is the software Bozorth3 developed by the National Institute of Standard and Technologies (NIST) [18].

- Features related to the shape of the ROI $\left(F_{S}\right)$ : this set is composed by 3 features that permit to evaluate the presence of deformations caused by errors in the estimation of the finger volume. These features are the length and width of the ROI, and the eccentricity of the ROI shape.

- Gabor features $\left(F_{G}\right)$ : this set is composed by $m_{G} \times$ $n_{G} \times \theta_{G}$ values. Starting from the image $I, \theta_{G}$ images $G_{\theta}$ are computed by applying a set of $\theta_{G}$ Gabor filters with different orientations. Each image $G_{\theta}$ is then divided into $m_{G} \times n_{G}$ local regions, and the absolute average distance (AAD) of the intensity values is computed for each region. In the spatial domain, a symmetric Gabor filter can be described as

$$
\begin{aligned}
G(x, y ; f, \theta) & =\exp \left\{-\frac{1}{2}\left[\frac{x^{\prime 2}}{\sigma_{x^{\prime}}^{2^{\prime}}}+\frac{y^{\prime 2}}{\sigma_{y^{\prime}}^{2^{\prime}}}\right]\right\} \cos 2 \pi f x^{\prime} \\
x^{\prime} & =x \sin \theta+y \cos \theta \\
y^{\prime} & =x \cos \theta-y \sin \theta
\end{aligned}
$$

where $f$ is the frequency of the sinusoidal plane wave along the direction $\theta$ from the $x$-axis, and $\sigma_{x}^{\prime}$ and $\sigma_{y}^{\prime}$ are the space constants of the Gaussian envelope along the $x_{0}$ and $y_{0}$ axes, respectively.

- Hog Features $\left(F_{H}\right)$ : this set is composed by $c_{w} \times c_{h} \times c_{b}$ features. These values are obtained by computing a set of Histogram of Oriented Gradients (HOG) features in the area of the image $I$ appertaining to the ROI. The HOG features are designed for general object recognition systems. We computed these features by applying the algorithm described in [20]. The first step of this algorithm is the computation of the gradient module image $G_{M}(x, y)$ and the gradient phase image $G_{P}(x, y)$ of the image $I$. Then, these two images are divided into $c_{w} \times c_{h}$ local regions. At each cell, the orientation $\tilde{G}_{P}(x, y)$ is then quantized into $c_{b}$ orientation bins, and weighted by its magnitude $G_{M}(x, y)$. Finally, the histogram with the $c_{b}$ orientations is computed for each cell.

- Standard deviation of Gabor features $\left(F_{G \sigma}\right)$ : this set is composed by $m_{G} \times n_{G}$ features. Similarly to the Gabor features, $\theta_{G}$ images $G_{\theta}$ are obtained by applying a set of $\theta_{G}$ Gabor filters with different orientations to the image $I$. Each image $G_{\theta}$ is then divided in $m_{G} \times n_{G}$ local regions and the absolute average distance of the intensity values of each region is computed. For each local region, the standard deviation of the values obtained by applying the $\theta_{G}$ Gabor filters is finally computed. These features permit to evaluate the presence of information in the local regions of the image, reducing the number of used values with respect to the Gabor features.

- Standard deviation of Gabor features $\left(F_{H \sigma}\right)$ : this set is composed by $c_{w} \times c_{h}$ features. A set of HOG features are computed by dividing the image $I$ in $c_{w} \times c_{h}$ local areas. For each local region, the standard deviation of the $c_{b}$ obtained HOG values is finally computed. The size of this feature set is lower than the size of the set of HOG features $F_{H}$.

\section{Quality estimation}

The quality estimation aims to distinguish the unwrapped fingerprint images that can be properly used to perform an identity comparison and the images affected by artifacts due to the presence of noise in the corresponding three-dimensional models. We consider the quality estimation as a classification problem. This task is performed by assigning two different classes to the unwrapped fingerprint images: "sufficient" and "poor". In this work, we use classifiers based on feedforward neural networks.

\section{EXPERIMENTAL RESULTS}

We tested the proposed method by using a set of 300 unwrapped fingerprint images with a resolution of about 500 ppi. These images are captured from 30 different individuals (10 images per individual). The unwrapped fingerprint images are related to the three-dimensional models computed by using the reconstruction system described in [1]. The input pairs of images captured by the two-view acquisition system are affected by different kinds of problems: reflections, out of focus, and dirty fingers. These problems compromised the correctness of some reconstructed three-dimensional models and, consequently, the quality of the corresponding unwrapped images. The obtained unwrapped images can present deformations, artifacts, and areas with low visibility of the ridge pattern. The proposed method aims to detect the presence of the mentioned problems in order to provide a qualitative measure of the unwrapped fingerprint images, which can be used to improve the accuracy of the biometric recognition process.

The acquisition setup is shown in Fig. 4 and is composed by two synchronized Sony XCD-SX90CR CCD color camera, and a DLP projector. The parameters of the setup are: $\alpha=85^{\circ}$, $\Delta_{D}=45 \mathrm{~mm}, \Delta_{P}=460 \mathrm{~mm}, \Delta_{H}=205 \mathrm{~mm}$. The two-view system is calibrated by using the technique described in [21, 22], with 15 pairs of chessboard images captured in different positions. The used calibration chessboard is composed by $12 \times 9$ squares of $2.8 \times 2.8 \mathrm{~mm}$.

We assigned a quality value for each unwrapped fingerprint image. Similarly to the approach described in [17], this value is a predictor of a matcher's performance. The first step of the estimation consists in the computation of the distribution of genuines and impostors by using the identity comparison 


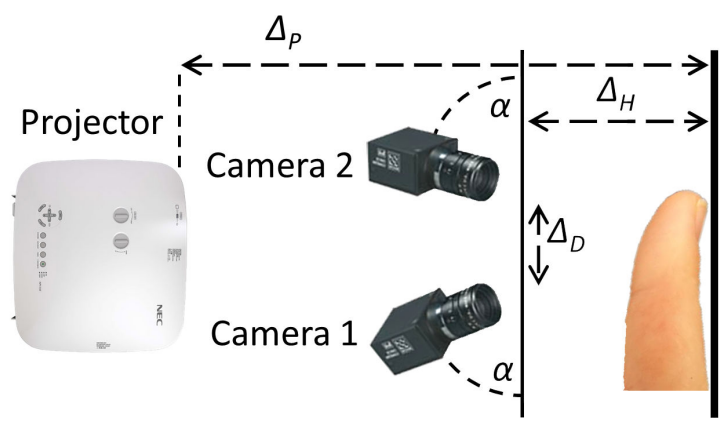

Fig. 4. Schema of the acquisition setup.

software NIST Bozorth3 [18]. Considering the obtained results, we define a metric called normalized match-score in order to evaluate the capability of a sample to be properly matched with other samples of the same individual. This measure permits to evaluate if the biometric sample contains sufficient information, and is defined as

$$
o\left(x_{i}\right)=s_{m}\left(x_{i i}\right)+\left(\mu\left(s_{m}\left(x_{i j}\right)\right)-s_{m}\left(x_{i i}\right)\right) / \sigma\left(s_{m}\left(x_{i j}\right)\right),
$$

where $x_{i}$ is the considered sample, $s_{m}\left(x_{i i}\right)$ is the matchscore obtained by comparing the sample $x_{i}$ with $x_{i}$ itself, $\mu\left(s_{m}\left(x_{i j}\right)\right)$ and $\sigma\left(s_{m}\left(x_{i j}\right)\right)$ are the mean and standard deviation of the match-scores obtained by comparing the sample $x_{i}$ with the other samples appertaining to the same individual. In order to maximize the distance between genuines and impostors, we define the class of each sample $x_{i}$ as

$$
q\left(x_{i}\right)=\left\{\begin{array}{ll}
+1 & \text { if } o\left(x_{i}\right)>96 \\
-1 & \text { otherwise }
\end{array} .\right.
$$

The value +1 corresponds to the class "sufficient", and the value -1 corresponds to the class "poor". The resulting number of "sufficient" images is 244 and the number of "poor" images is 56 .

Fig. 5 shows an example of an unwrapped fingerprint image with sufficient quality and two examples of poor quality images. The image in Fig. 5(b) suffers of deformations related to the presence of improperly reconstructed regions of the corresponding three-dimensional model. Differently, Fig. 5(c) suffers of artifacts caused by out of focus regions in the pair of images captured using the two-view acquisition system presented in [1].

\section{A. Classification results}

We experimentally evaluated different values of the parameters used by the feature extraction algorithms. In order to compute the Gabor features, we used three different sets of angles $\theta$ :

- $\Theta_{a}=\left(0^{\circ}, 90^{\circ}\right)$,

- $\Theta_{b}=\left(-45^{\circ}, 45^{\circ}\right)$,

- $\Theta_{c}=\left(-45^{\circ}, 0^{\circ}, 45^{\circ}, 90^{\circ}\right)$.

We also evaluated values from 1 to 6 for the parameters $m_{G}$ and $n_{G}$. Similiarly, we considered values from 1 to 6 for the parameters $c_{w}$ and $c_{h}$ describing the number of local regions (a)

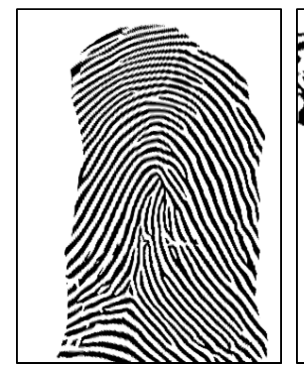

Fig. 5. Examples of classified unwrapped fingerprint images: (a) sufficient quality image; (b) poor quality image with shape deformations; (c) poor quality image affected by the presence of artifacts.

TABLE I

\begin{tabular}{|c|c|c|c|c|c|c|c|c|}
\hline \multirow{2}{*}{$\begin{array}{c}\text { Feature Set } \\
\text { Name }\end{array}$} & \multicolumn{8}{|c|}{ Feature Set Composition } \\
\hline & Minutiae & e ROI & $\begin{array}{l}F_{G}, \\
\Theta_{a}\end{array}$ & $\begin{array}{l}F_{G}, \\
\Theta_{b}\end{array}$ & $\begin{array}{l}F_{G}, \\
\Theta_{c}\end{array}$ & $F_{H}$ & $\begin{array}{l}F_{G \sigma} \\
\Theta_{c}\end{array}$ & $F_{H o}$ \\
\hline Gabor-a1 & & & $\sqrt{ }$ & & & & & \\
\hline Gabor-a2 & $\sqrt{ }$ & & $\sqrt{ }$ & & & & & \\
\hline Gabor-a3 & & $\sqrt{ }$ & $\sqrt{ }$ & & & & & \\
\hline Gabor-a4 & $\sqrt{ }$ & $\sqrt{ }$ & $\sqrt{ }$ & & & & & \\
\hline Gabor-b1 & & & & $\sqrt{ }$ & & & & \\
\hline Gabor-b2 & $\sqrt{ }$ & & & $\sqrt{ }$ & & & & \\
\hline Gabor-b3 & $\sqrt{ }$ & $\sqrt{ }$ & & $\sqrt{v}$ & & & & \\
\hline $\begin{array}{l}\text { Gabor-b4 } \\
\text { Gabor-c1 }\end{array}$ & & & & & $\sqrt{ }$ & & & \\
\hline Gabor-c2 & $\sqrt{ }$ & & & & $\sqrt{ }$ & & & \\
\hline Gabor-c3 & & $\sqrt{ }$ & & & $\sqrt{ }$ & & & \\
\hline Gabor-c4 & $\sqrt{ }$ & $\sqrt{ }$ & & & $\sqrt{ }$ & & & \\
\hline HOG-1 & & & & & & $\sqrt{ }$ & & \\
\hline HOG-2 & $\sqrt{ }$ & & & & & $\sqrt{ }$ & & \\
\hline HOG-3 & & $\sqrt{ }$ & & & & $\sqrt{ }$ & & \\
\hline HOG-4 & $\sqrt{ }$ & $\sqrt{ }$ & & & & $\sqrt{ }$ & & \\
\hline Gabor-std-1 & & & & & & & $\sqrt{ }$ & \\
\hline Gabor-std-2 & $\sqrt{ }$ & & & & & & $\sqrt{ }$ & \\
\hline Gabor-std-3 & & $\sqrt{ }$ & & & & & $\sqrt{ }$ & \\
\hline Gabor-std-4 & $\sqrt{ }$ & $\sqrt{ }$ & & & & & $\sqrt{ }$ & \\
\hline HOG-std-1 & & & & & & & & $\sqrt{ }$ \\
\hline HOG-std-2 & $\sqrt{ }$ & & & & & & & $\sqrt{ }$ \\
\hline HOG-std-3 & & $\sqrt{ }$ & & & & & & $\sqrt{ }$ \\
\hline HOG-std-4 & $\sqrt{ }$ & $\sqrt{ }$ & & & & & & $\sqrt{ }$ \\
\hline
\end{tabular}

FeATURE DATASETS.

used during the computation of the HOG features, and values from 3 to 12 for the parameters $c_{b}$, which define the number of considered orientation bins.

Considering different combinations of features, we created 24 different feature sets, which are summarized in Table I.

The quality evaluation of the feature sets is performed by using classifiers based on feedforward neural networks. The topology of the neural networks has been designed as follows: we used a linear node as output layer for the neural networks and we tested different numbers of nodes in the hidden layer. The nodes of the hidden layer are tan-sigmoidal. The method used for the training of the neural networks is the backpropagation algorithm. In order to properly estimate the generalization capability of the trained neural networks, we used a $\mathrm{N}$-fold cross validation technique with $\mathrm{N}=10$ [23].

Table II reports the results obtained by the neural classifiers on the evaluated feature sets. This table shows only the best configurations found during the analysis, describing the parameters used by the feature extraction algorithms, the number of considered features, and the number of nodes of the 
TABLE III

RESULTS OF DIFFERENT CLASSIFIERS ON THE BEST FEATURE SET (GABOR-STD-1).

\begin{tabular}{ccc}
\hline \hline Classifier & Total & Std \\
\hline FNN-45 & 0.01 & 0.04 \\
ldc & 0.18 & 0.013 \\
klldc & 0.18 & 0.011 \\
pcldc & 0.18 & 0.006 \\
quad & 0.17 & 0.002 \\
kNN-1 & 0.16 & 0.007 \\
kNN-3 & 0.16 & 0.003 \\
kNN-5 & 0.15 & 0.007 \\
kNN-10 & 0.16 & 0.002 \\
\hline \hline
\end{tabular}

Notes: Total $=$ total classification error; Std $=$ standard deviation of the classification error; NN-45 $=$ Feedforward Neural Network with one hidden layer composed by 45 nodes; lin = Linear classifier; klldc $=$ Linear Classifier using KL expansion; pcldc $=$ Linear Classifier using PC expansion; quad $=$ Quadratic classifier; $\mathrm{kNN}=\mathrm{k}$ Nearest Neighbor, where $\mathrm{k}$ stands for the number of first neighbors.

hidden layer. Table II shows that the proposed method obtained a remarkable accuracy with all the evaluated feature sets. Moreover, the best configurations obtained a total classification error equal to the $1 \%$. It is also possible to observe that the features computed by using Gabor Filters $\left(F_{G}\right.$ and $\left.F_{G \sigma}\right)$ are the most discriminative ones. Another interesting observation is that the accuracy obtained by using the feature sets $F_{G}$ and $F_{H}$ is very similar to the accuracy obtained by using $F_{G \sigma}$ and $F_{G \sigma}$.

We compared the results obtained by different classifiers on the best feature set. Table III shows the results obtained by applying feedforward neural networks, k Nearest Neighbor, linear, and quadratic classifiers on the feature set Gabor-std-1 (with $m_{G}=6, n_{G}=6$, and $\theta_{G}=4$ ).

Table III shows that simple classifiers do not obtain sufficient results on the evaluated dataset. Differently, classifiers able to approximate more complex functions, like neural networks with a large number of hidden neurons, can obtain a signification reduction of the classification error. For example, in our experiments, with 45 hidden neurons, the mean error had been reduced by a factor of 10 with respect to different kinds of simpler classifiers.

\section{B. Comparison with literature methods}

The application of the reference software for quality classification of fingerprint images NFIQ developed by the National Institute of Standard and Technologies [17, 18] on the studied dataset of unwrapped fingerprint images is reported in Fig. 5, where the obtained class distribution is plotted.

It is worth noting that the NFIQ software is designed for the quality classification of fingerprint images captured using touch-based sensors and its application in the context of touchless fingerprint images produces sufficient results.

In order to compare the performances of the two methods, we performed an identification test by using the identity comparison method NIST Bozorth3 [18] and by discarding the images classified as insufficient by applying the two considered methods. Table IV reports the number of discarded

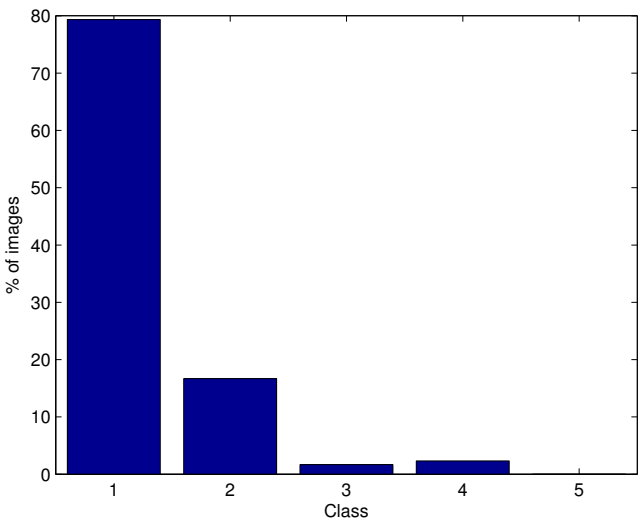

Fig. 6. Class distribution of the reference software NIST NFIQ on the studied dataset.

TABLE IV

EFFECTS ON THE EER OF THE APPLICATION OF DIFFERENT QUALITY CLASSIFIERS FOR THE STUDIED DATASET OF UNWRAPPED FINGERPRINT IMAGES.

\begin{tabular}{cccc}
\hline \hline Data & $\begin{array}{c}\text { Used } \\
\text { samples }\end{array}$ & $\begin{array}{c}\text { Discarded } \\
\text { samples }\end{array}$ & EER (\%) \\
\hline Original dataset & 300 & 0 & 9.56 \\
$q_{N F I Q}=1$ & 238 & 62 & 5.76 \\
$q_{N F I Q}<=2$ & 288 & 12 & 7.45 \\
Proposed method & 244 & 56 & 1.97 \\
\hline \hline
\end{tabular}

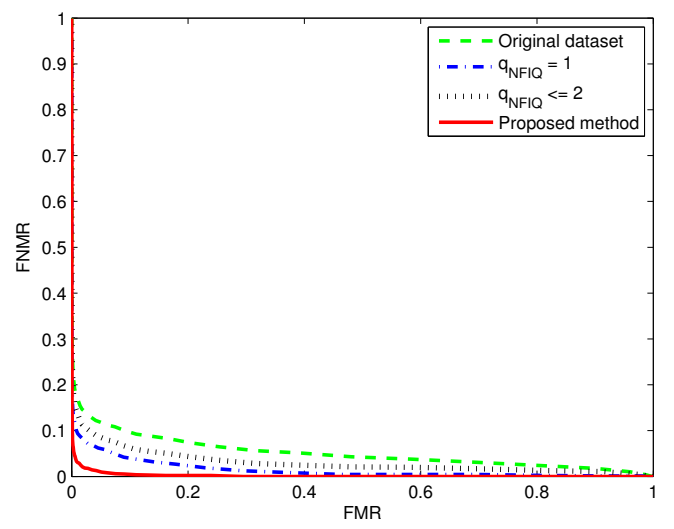

Fig. 7. Effects on the ROC curves of the application of the proposed method and the software NIST NFIQ on the test dataset composed by 300 unwrapped images of fingertip three-dimensional models.

samples and the obtained equal error rate (EER). Fig. 7 shows the obtained Receiver operating characteristic (ROC) curves.

Table IV and Fig. 7 show that the proposed method effectively improved the accuracy of the biometric system on the evaluated dataset. In fact, the EER obtained without using the proposed approach is equal to the $9.56 \%$, and the EER obtained by discarding the "poor" quality images estimated by the proposed approach is the $1.97 \%$. The results obtained on the test dataset by the proposed approach are also better than the ones obtained by the method NIST NFIQ. The proposed approach, in fact, excluded a minor number of samples (56 
TABLE II

QUALITY CLASSIFICATION ACCURACY OF NEURAL CLASSIFIERS WITH DIFFERENT FEATURE SETS.

\begin{tabular}{|c|c|c|c|c|c|c|c|c|c|c|}
\hline Feature Set & Parameters & Features \# & Fidden & TP (\%) & FN (\%) & FP (\%) & TN (\%) & Sensitivity & Specificity & Total (\%) \\
\hline Gabor-a1 & $m_{G}=3, n_{G}=3, \theta_{G}=2$ & 18 & 60 & 18.00 & 0.67 & 2.33 & 79.00 & 0.96 & 0.97 & 3.00 \\
\hline Gabor-a2 & $m_{G}=5, n_{G}=5, \theta_{G}=2$ & 55 & 35 & 18.00 & 0.67 & 0.67 & 80.67 & 0.96 & 0.99 & 1.33 \\
\hline Gabor-a3 & $m_{G}=4, n_{G}=4, \theta_{G}=2$ & 36 & 45 & 17.67 & 1.00 & 1.00 & 80.33 & 0.95 & 0.99 & 2.00 \\
\hline Gabor-a4 & $m_{G}=4, n_{G}=4, \theta_{G}=2$ & 39 & 75 & 17.67 & 1.00 & 1.00 & 80.33 & 0.95 & 0.99 & 2.00 \\
\hline Gabor-b1 & $m_{G}=4, n_{G}=4, \theta_{G}=2$ & 32 & 70 & 17.00 & 1.67 & 0.67 & 80.67 & 0.91 & 0.99 & 2.33 \\
\hline Gabor-b2 & $m_{G}=5, n_{G}=5, \theta_{G}=2$ & 55 & 55 & 17.67 & 1.00 & 0.33 & 81.00 & 0.95 & 1.00 & 1.33 \\
\hline Gabor-b3 & $m_{G}=4, n_{G}=4, \theta_{G}=2$ & 36 & 70 & 17.67 & 1.00 & 2.00 & 79.33 & 0.95 & 0.98 & 3.00 \\
\hline Gabor-b4 & $m_{G}=5, n_{G}=5, \theta_{G}=2$ & 57 & 75 & 17.33 & 1.33 & 1.00 & 80.33 & 0.93 & 0.99 & 2.33 \\
\hline Gabor-c1 & $m_{G}=3, n_{G}=3, \theta_{G}=4$ & 36 & 50 & 16.67 & 2.00 & 0.33 & 81.00 & 0.89 & 1.00 & 2.33 \\
\hline Gabor-c2 & $m_{G}=5, n_{G}=5, \theta_{G}=4$ & 67 & 75 & 18.33 & 0.33 & 0.67 & 80.67 & 0.98 & 0.99 & 1.00 \\
\hline Gabor-c3 & $m_{G}=4, n_{G}=4, \theta_{G}=4$ & 68 & 55 & 16.33 & 2.33 & 0.67 & 80.67 & 0.88 & 0.99 & 3.00 \\
\hline Gabor-c4 & $m_{G}=4, n_{G}=4, \theta_{G}=4$ & 71 & 30 & 17.67 & 1.00 & 0.33 & 81.00 & 0.95 & 1.00 & 1.33 \\
\hline HOG-1 & $c_{w}=3, c_{h}=3, c_{b}=12$ & 108 & 55 & 16.67 & 2.00 & 1.00 & 80.33 & 0.89 & 0.99 & 3.00 \\
\hline HOG-2 & $c_{w}=3, c_{h}=3, c_{b}=9$ & 86 & 35 & 17.00 & 1.67 & 0.33 & 81.00 & 0.91 & 1.00 & 2.00 \\
\hline HOG-3 & $c_{w}=3, c_{h}=3, c_{b}=9$ & 85 & 55 & 16.00 & 2.67 & 0.33 & 81.00 & 0.86 & 1.00 & 3.00 \\
\hline HOG-4 & $c_{w}=3, c_{h}=3, c_{b}=9$ & 88 & 50 & 17.33 & 1.33 & 1.00 & 80.33 & 0.93 & 0.99 & 2.33 \\
\hline Gabor-std-1 & $m_{G}=6, n_{G}=6, \theta_{G}=4$ & 36 & 45 & 18.00 & 0.67 & 0.33 & 81.00 & 0.96 & 1.00 & 1.00 \\
\hline Gabor-std-2 & $m_{G}=4, n_{G}=4, \theta_{G}=4$ & 20 & 65 & 17.00 & 1.67 & 0.33 & 81.00 & 0.91 & 1.00 & 2.00 \\
\hline Gabor-std-3 & $m_{G}=6, n_{G}=6, \theta_{G}=4$ & 39 & 40 & 17.33 & 1.33 & 0.33 & 81.00 & 0.93 & 1.00 & 1.67 \\
\hline Gabor-std-4 & $m_{G}=5, n_{G}=5, \theta_{G}=4$ & 32 & 60 & 17.00 & 1.67 & 0.00 & 81.33 & 0.91 & 1.00 & 1.67 \\
\hline HOG-std-1 & $c_{w}=3, c_{h}=3, c_{b}=9$ & 9 & 55 & 14.67 & 4.00 & 0.33 & 81.00 & 0.79 & 1.00 & 4.33 \\
\hline HOG-std-2 & $c_{w}=3, c_{h}=3, c_{b}=9$ & 13 & 55 & 17.33 & 1.33 & 2.00 & 79.33 & 0.93 & 0.98 & 3.33 \\
\hline HOG-std-3 & $c_{w}=3, c_{h}=3, c_{b}=9$ & 12 & 80 & 18.00 & 0.67 & 2.00 & 79.33 & 0.96 & 0.98 & 2.67 \\
\hline HOG-std-4 & $c_{w}=3, c_{h}=3, c_{b}=9$ & 16 & 70 & 16.33 & 2.33 & 1.33 & 80.00 & 0.88 & 0.98 & 3.67 \\
\hline
\end{tabular}

Notes: Features \# = number of features; Hidden = number of hidden layer nodes of the feedforward neural networks; TP = true positives; FN = false negatives; FP = false positives; $\mathrm{TN}=$ true negatives; Total $=$ total classification error.

v.s. 62) and obtained a better EER (1.97\% v.s. $5.76 \%)$. Moreover, experiments show that the recognition accuracy is enhanced with respect to the reference methods for almost all the ROC curve plot. This fact can be explained by considering that the kind of problems which can affect fingerprint images captured by touch-based sensors are different from the ones that can affect fingerprint images obtained by unwrapping three-dimensional finger models. Notably, the fact that the overall accuracy of the biometric system can be considered as lower than the state-of-the-art systems based on touchbased sensors can be related to the particularly noisy biometric samples that we used for testing the proposed approach.

\section{CONCLUSiON}

This paper presented an approach for the quality classification of fingerprint images obtained by performing the unwrapping of three-dimensional fingertip models. Methods in the literature designed for fingerprint images captured by touch-based sensors are not adequate for this kind of images. Unwrapped fingerprint images, in fact, need to be checked for their quality level taking into account also new non-idealities that are not typically present in traditional fingerprint images. For example, badly reconstructed regions and spikes in the three-dimensional models can produce important deformations of the ridge pattern in the unwrapped images and the injection of new kinds of image noise. Badly reconstructed regions or spikes in the three-dimensional models, for example, can produce important deformations of the ridge pattern in the unwrapped images.

The paper presented different sets of features that can be used for the quality estimation of the evaluated fingerprint images and it describes the designing and training of the final neural classification system. Experimental results showed that the proposed classification method is achievable and it offers a suitable quality classification accuracy. The performances of the proposed approach were also evaluated in a complete biometric system and compared with a well-known method in the literature. The accuracy of the recognition system was effectively increased by using the proposed quality estimation approach and the results obtained on the test dataset are better than the ones obtained by the reference method. Further studies will be focused on the optimization of the computational complexity of the method as well as on the study of images obtained by using different three-dimensional reconstruction systems.

\section{REFERENCES}

[1] R. Donida Labati, A. Genovese, V. Piuri, and F. Scotti, "Fast 3-d fingertip reconstruction using a single two-view structured light acquisition," in IEEE Workshop on Biometric Measurements and Systems for Security and Medical Applications (BIOMS), September 2011, pp. 1 - 8.

[2] F. Alonso-Fernandez, J. Fierrez, J. Ortega-Garcia, J. GonzalezRodriguez, H. Fronthaler, K. Kollreider, and J. Bigun, "A comparative study of fingerprint image-quality estimation methods," IEEE Transactions on Information Forensics and Security, vol. 2, no. 4, pp. $734-$ 743, December 2007.

[3] R. Donida Labati, V. Piuri, and F. Scotti, "Neural-based quality measurement of fingerprint images in contactless biometric systems," in The 2010 International Joint Conference on Neural Networks (IJCNN), July 2010, pp. $1-8$.

[4] G. Parziale, "Touchless fingerprinting technology," Advances in Biometrics, pp. 39-62, 2007.

[5] G. Parziale, E. Diaz-Santana, and R. Hauke, "The Surround Imager ${ }^{\mathrm{TM}}$. A multi-camera touchless device to acquire $3 \mathrm{~d}$ rolled-equivalent fingerprints," Lecture Notes in Computer Science, vol. 3832, pp. 244-250, 2005.

[6] Y. Wang, L. G. Hassebrook, and D. L. Lau, "Data acquisition and processing of 3-D fingerprints," IEEE Transactions on Information Forensics and Security, vol. 5, no. 4, pp. 750-760, December 2010.

[7] — , "Noncontact, depth-detailed 3D fingerprinting," SPIE Newsroom, November 2009. 
[8] Y. Chen, G. Parziale, E. Diaz-Santana, and A. Jain, "3D touchless fingerprints: Compatibility with legacy rolled images," 2006 Biometrics Symposium, 2006.

[9] Y. Wang, L. G. Hassebrook, and D. L. Lau, "Fit-sphere unwrapping and performance analysis of 3D fingerprints," Applied Optics, vol. 49, no. 4, pp. $592-600,2010$

[10] L. G. H. Sara Shafaei, Tamer Inanc, "A new approach to unwrap a 3-D fingerprint to a 2-D rolled equivalent fingerprint," IEEE 3rd International Conference on Biometrics: Theory, Applications, and Systems, 2009. (BTAS'09), pp. 1-5, September 2009.

[11] Q. Zhao, A. Jain, and G. Abramovich, "3d to 2d fingerprints: Unrolling and distortion correction," in International Joint Conference on Biometrics (IJCB), October 2011, pp. $1-8$.

[12] S. Lee, H. seung Choi, K. Choi, and J. Kim, "Fingerprint-quality index using gradient components," IEEE Transactions on Information Forensics and Security, vol. 3, no. 4, pp. 792-800, 2008.

[13] L. Shen, A. C. Kot, and W. M. Koo, "Quality measures of fingerprint images," in AVBPA, 2001, pp. 266-271.

[14] N.Ratha and R.Bolle, "Fingerprint image quality estimation," IBM Computer Science Research, Report RC21622, 1999.

[15] D. Yu, L. Ma, H. Lu, and Z. Chen, "Fusion method of fingerprint quality evaluation: From the local gabor feature to the global spatial-frequency structures," 2006, pp. 776-785.

[16] X. kun Yang and Y. Luo, "A classification method of fingerprint quality based on neural network," in International Conference on Multimedia Technology (ICMT), July 2011, pp. $20-23$.

[17] E. Tabassi, C. Wilson, and C. Watson, "Fingerprint image quality," National Institute of Standards and Technology (NIST), Technical Report NISTIR 7151, August 2004.

[18] C. I. Watson, M. D. Garris, E. Tabassi, C. L. Wilson, R. M. McCabe, S. Janet, and K. Ko, "User's guide to nist biometric image software (NBIS)," National Institute of Standards and Technology, January 2007, http://fingerprint.nist.gov/NFIS.

[19] R. Donida Labati, V. Piuri, and F. Scotti, "A neural-based minutiae pair identification method for touch-less fingerprint images," in IEEE Workshop on Computational Intelligence in Biometrics and Identity Management (CIBIM), April 2011, pp. 96 -102.

[20] D. G. Lowe, "Distinctive image features from scale-invariant keypoints," International Joint Conference on Computer Vision, vol. 60, pp. 91 110, November 2004.

[21] Z. Zhang, "A flexible new technique for camera calibration," IEEE Transactions on Pattern Analysis and Machine Intelligence, vol. 22, no. 11, pp. 1330-1334, 2000.

[22] J. Heikkila and O. Silvén, "A four-step camera calibration procedure with implicit image correction," IEEE Computer Society Conference on Computer Vision and Pattern Recognition (CVPR'97), pp. 1106-1112, 1997.

[23] R. O. Duda, P. E. Hart, and D. G. Stork, Pattern Classification (2nd Edition), 2nd ed. Wiley-Interscience, November 2000 